\title{
Occurrence of Extended-Spectrum Beta-lactamases in Enterobacteriaceae Isolated from Hospitalized Patients in Curitiba, southern Brazil
}

\author{
Keite da Silva Nogueira ${ }^{1,2}$, Ilma Hiroko Higuti ${ }^{2,3}$, \\ Agnaldo José do Nascimento ${ }^{4}$, Larissa Bail Terasawa ${ }^{3}$, \\ Simone de Oliveira ${ }^{3}$, Adriana Pereira Matos ${ }^{5,6}$, \\ Helena Aguilar Peres Homem de Mello de Souza ${ }^{1}$, \\ Laura Lúcia Cogo ${ }^{1,2}$ and Libera Maria Dalla Costa ${ }^{1,3,6}$
}

\begin{abstract}
${ }^{1}$ Laboratory of Bacteriology, Federal University of Paraná, ${ }^{2}$ Department of Basic Pathology, Federal University of Paraná, ${ }^{3}$ Program of Post-graduation in Microbiology, Parasitology and Pathology, Federal University of Paraná, ${ }^{4}$ Department of Biochemistry, Federal University of Paraná, ${ }^{5}$ Hospital Cajuru - Catholic University of Paraná, ${ }^{6}$ Program of Post-graduation in Pharmaceutical Science, Federal University of Paraná; Curitiba, PR, Brazil
\end{abstract}

\begin{abstract}
Production of extended-spectrum beta-lactamases (ESBL) by enterobacteria is an important resistance mechanism against antimicrobial beta-lactamics. We tested 498 bacterial strains isolated from two tertiary-care teaching hospitals for ESBL production, using screening breakpoints for aztreonam and third generation cephalosporins, according to CLSI recommendations. Among these isolates, 155 were positive for the ESBL screening test, and $121(78 \%)$ were confirmed by the clavulanic acid combination disk method. We found a high frequency of ESBL (24\%) among Enterobacteriaceae, with a frequency of $\mathbf{5 7 . 4 \%}$ for Klebsiella pneumoniae, $\mathbf{2 1 . 4 \%}$ for Klebsiella oxytoca, and $\mathbf{7 . 2 \%}$ for $\boldsymbol{E}$. coli. In other members of Enterobacteriaceae, non-Klebsiella and non-E. coli, the prevalence was $\mathbf{2 1 . 6 \%}$. Ceftriaxone and cefotaxime showed a higher sensitivity in the screening test $(99.2 \%)$ when compared to ceftazidime, aztreonam and cefpodoxime. However, cefotaxime/cefotaxime plus clavulanic acid showed a higher sensitivity in the confirmatory test $(96.7 \%)$.

Key Words: ESBL, beta-lactamases, Enterobacteriaceae.
\end{abstract}

Extended-spectrum beta-lactamases (ESBL) were initially described by Knothe et al. (1983) [1]; they have contributed to the recently-observed large increase in beta-lactamic antimicrobial resistance. These enzymes are capable of hydrolyzing penicillins, cephalosporins (including wide spectrum ones) and monobactamics, except for the cefamicines and the carbapenemics [2]. The ESBLs are sensitive to inhibitors of beta-lactamases, such as clavulanic acid, classified in group 2 of Bush et al. [3].

Many of the ESBLs arise from point mutations in genes that encode narrow-spectrum beta-lactamases, provided by plasmids such as TEM and SHV [2]. However, members of a newly-emerging ESBL group, CTX-M, derived from class A chromosomal beta-lactamases, have been identified recently [4]. Routine antimicrobial susceptibility tests do not always detect ESBLs, thus requiring special detection tests [5].

Plasmid genes are easily transferred among enterobacteria, contributing to ESBL dissemination [6]. Plasmids that carry beta-lactamase genes frequently harbor

Received on 8 June 2006; revised 14 November 2006.

Address for correspondence: Dr. Libera Maria Dalla Costa. Hospital de Clínicas - Universidade Federal do Paraná, Laboratório de Bacteriologia. Rua Padre Camargo, 280, $1^{\circ}$ Andar, Sala 203. Zip code: 80.060-240 - Curitiba, Paraná, Brazil. Phone-55-41-33607975 / Fax -55-41-3360-1811. E-mail:1mdc@ufpr.br.

The Brazilian Journal of Infectious Diseases 2006;10(6):390-395. (C) 2006 by The Brazilian Journal of Infectious Diseases and Contexto Publishing. All rights reserved. resistance genes to other antimicrobials, such as trimethopim-sulfamethoxazole and aminoglycosides [7]. Therefore, the detection of ESBL-producing isolates is critical to assure appropriate therapy and to provide necessary information to prevent their dissemination.

The Clinical Standard Laboratory Institute (CLSI) recommends that screening and confirmatory tests should be routinely undertaken for $K$. pneumoniae, $K$. oxytoca, E. coli and Proteus mirabilis. The screening tests are based on the detection of an increase in the minimum inhibitory concentration (MIC) or a reduction in the susceptibility zone diameter for one or more of five antimicrobial agents (cefpodoxime, aztreonam, ceftriaxone, cefotaxime and ceftazidime). Confirmatory tests are based on the increased susceptibility to antimicrobial agents tested in combination with clavulanic acid versus susceptibility when tested alone.

According to Schawber et al. [8], the identification of ESBLs in non-E.coli and non-Klebsiella spp. enterobacteria and the applicability of the CLSI criteria for their detection remain uncertain and inconclusive. Proteus mirabilis was the first non-classic ESBLproducing species included in CLSI 2005 for screening and confirmatory tests of isolates from clinically-relevant samples (e.g., blood samples).

We analyzed the occurrence of ESBL in different enterobacteria species and determined the best enzyme substrate to detect ESBL in two university hospitals. We also studied the pattern of susceptibility of the isolates to other clinically-relevant antimicrobials. 


\section{Material and Methods}

$\underline{\text { Samples }}$

Four-hundred-ninety-eight isolates of enterobacteria were obtained prospectively from hospitalized patients. The strains were derived from urine, blood, cerebrospinal fluid, sputum, tracheal aspirate, bronchoalveolar lavage and surgical wounds. Among these, 438 isolates were obtained from the Clinical Hospital of the Federal University of Paraná (HCUFPR) from August 2003 to August 2004. Sixty isolates were obtained from patients at the Hospital of the Catholic University of Paraná (HUC-PUC), from January to August 2004. The preliminary identification of Enterobacteriaceae was accomplished using the Vitek @ (bioMérieux) automated system and afterwards confirmed by a panel of standard biochemical tests recommended for enterobacteria identification [9-14].

Disk-Diffusion Screening (DD) and ESBL confirmatory test

The disk-diffusion screening and ESBL confirmatory test was performed as described by Clinical Laboratory Standard International $[14,15]$.

Determination of the Minimum Inhibitory Concentration (MIC)

A MIC assay was performed on all strains that showed zone reduction for one or more of the antimicrobials used in the ESBL screening test. The agar micro-dilution technique was performed as described by the National Committee for Clinical Laboratory Standard [16], using amikacin, aztreonam, cefepime, cefotaxime, ceftazidime and ceftriaxone (Sigma Chemical CO, St. Louis, MO, USA), ciprofloxacin (ICN Biomedicals Inc. Dawn, OH, USA), imipenem and ertapenem (Merck Sharp \& Dohme Ltda., Campinas, SP, Brazil). Pseudomonas aeruginosa ATCC 27853 and E. coli ATCC 25922 [17] were used as quality-control strains.

\section{Results}

Among the 498 strains of enterobacteria that were isolated, 155 presented decreased susceptibility to third-generation cephalosporins and aztreonam and were considered possible ESBL producers. Among these, 121 isolates were confirmed by the combined disk method. Table 1 presents the number of isolates of each species and the distribution of positive samples by screening and confirmatory tests. The largest isolate numbers from species confirmed as ESBL positive were K. pneumoniae (67), E. cloacae (20) and E. coli (14). Serratia marcescens, Enterobacter aerogenes and $K$. oxytoca provided three or four confirmed bacterial isolates, while other species provided one or two, except for Enterobacter gergoviae, which did not provide any ESBL isolates.

The prevalence of ESBL-positive isolates within the group of enterobacteria was $24.3 \%$. Among the species considered classic ESBL producers, K. pneumoniae was the species with the highest isolate occurrence (57.4\%), followed by $K$. oxytoca (21.4\%), and E. coli $(7.2 \%)$, with a $21.6 \%$ positive frequency for other species isolates. Proteus mirabilis, which recently was included in the CLSI 2005 among species recommended for ESBL testing generated a positive isolate frequency of $8 \%$ in our study. By grouping the affected isolates by genus, a high positive percentage for Klebsiella (54.3\%), Enterobacter (27.1\%), and Serratia (20.9\%) was observed. The occurrence of Citrobacter, Morganella, Proteus and Escherichia was also striking, varying from $7.3 \%$ to $14.3 \%$. The genera Providencia and Pantoea were not included in this analysis, due to the small number of isolates. Among the positive enterobacteria identified by the screening test, 20 isolates of Enterobacter (23.5\%), five isolates of Klebsiella (4.0\%), three isolates of Serratia (12.5\%), three isolates of Morganella (17.6\%) and one isolate of Citrobacter (7.1\%) provided inconclusive results by the clavulanic acid inhibition test.

Sixty-four (52.9\%) of $121 \mathrm{ESBL}$ isolates were resistant to cefoxitine. The sum of the frequency of resistant samples to cefoxitine in non- $E$. coli and non-Klebsiella spp. species were higher than those found in classic ESBL producers. Among the classic producers, the highest frequency of resistance to cefoxitine was found in the K. pneumoniae isolates, followed by K.oxytoca and E.coli. Among non-E.coli and nonKlebsiella spp. isolates, E. cloacae had 18 of 20 ESBL positive isolates resistant to cefoxitine. Out of four ESBL-positive $S$. marcescens isolates, three were resistant to cefoxitine. The species $S$. liquefaciens, $P$. rettgerii, $P$. mirabilis, P.agglomerans, M. morganii, E. gergoviae, E. aerogenes, C.amalonaticus and $C$. freundii each included only one isolate resistant to cefoxitine as well as ESBL positive, while P. vulgaris had none.

The antimicrobials used for ESBL testing were analyzed for estimation of sensitivity and specificity in the detection of ESBL by comparing the isolates from the screen test with those positive in the confirmatory test. The greatest sensitivity was obtained with ceftriaxone and the smallest with ceftazidime. The specificity was low for all the antimicrobials (Table 2).

When only E. coli and Klebsiella spp. were analyzed, the results of sensitivity and specificity were similar, and the sensitivity to ceftazidime was lower for the ESBL detection of non-E. coli and non Klebsiella spp. species. Moreover, in the confirmatory test for the production of ESBL, a higher sensitivity was observed with cefotaxime (CTX) and cefotaxime-clavulanic acid (CTX+AC). The specificity was $100 \%$ for all combinations (Table 2). This was seen only when E. coli and Klebsiella spp. were evaluated. However, the sensitivity for all combinations of non-E.coli and nonKlebsiella spp. species was lower. When evaluating the two combinations used simultaneously, the sensitivity for cefpodoxime and cefpodoxime together with clavulanic acid $(\mathrm{CPD} / \mathrm{CPD}+\mathrm{AC})$ against ceftazidime and ceftazidime combined 
Table 1. Frequency of ESBL-positive screen and confirmatory tests distributed by species and genus

\begin{tabular}{|c|c|c|c|c|c|c|c|c|c|c|c|}
\hline Species & Isolate & $\mathbf{S T}(+)$ & CT (+) & Genus & Isolate & ST (+) & $\%$ & CI & CT (+) & $\%$ & CI \\
\hline Citrobacter amalonaticus & 1 & 1 & 1 & Citrobacter & 14 & 3 & 21.4 & $5.7-51.2$ & 2 & 14.3 & $2.5-43.8$ \\
\hline Citrobacter freundii & 13 & 2 & 1 & & & & & & & & \\
\hline Enterobacter aerogenes & 14 & 7 & 3 & Enterobacter & 85 & 43 & 50.6 & $39.6-61.5$ & 23 & 27.1 & 18.3-37.9 \\
\hline Enterobacter cloacae & 67 & 34 & 20 & & & & & & & & \\
\hline Enterobacter gergoviae & 4 & 2 & 0 & & & & & & & & \\
\hline Escherichia coli & 193 & 14 & 14 & Escherichia & 193 & 14 & 7.2 & $4.2-12.1$ & 14 & 7.3 & $4.2-12.1$ \\
\hline Klebsiella oxytoca & 14 & 3 & 3 & Klebsiella & 129 & 75 & 58.1 & $49.1-66.7$ & 70 & 54.3 & $45.3-63.0$ \\
\hline Klebsiella pneumoniae & 115 & 72 & 67 & & & & & & & & \\
\hline Morganella morgani & 17 & 5 & 2 & Morganella & 17 & 5 & 29.4 & $11.38-56.0$ & 2 & 11.8 & $2.1-37.7$ \\
\hline Pantoea agglomerans & 2 & 1 & 1 & Pantoea & 2 & 1 & * & * & 1 & * & * \\
\hline Proteus mirabilis & 25 & 4 & 2 & Proteus & 33 & 5 & 15.2 & $5.7-32.7$ & 3 & 9.1 & $2.3-25.5$ \\
\hline Proteus vulgaris & 8 & 1 & 1 & & & & & & & & \\
\hline Providencia rettgeriii & 1 & 1 & 1 & Providencia & 1 & 1 & * & * & 1 & * & $*$ \\
\hline Serratia liquefaciens & 4 & 1 & 1 & Serratia & 24 & 8 & 33.3 & $16.4-55.3$ & 5 & 20.9 & $7.9-42.7$ \\
\hline Serratia marcescens & 20 & 7 & 4 & & & & & & & & \\
\hline Total & 498 & 155 & 121 & & 498 & 155 & 31.1 & & 121 & 24.3 & \\
\hline
\end{tabular}

$\mathrm{ST}=$ screen test $\mathrm{CT}=$ confirmatory test $\mathrm{CI}=$ confidence interval.

Table 2. Sensitivity and specificity of the antimicrobials used for screening and confirmatory tests to detect ESBL producers

\begin{tabular}{llcc}
\hline Test & Antibiotics & Sensitivity (\%) & Specificity (\%) \\
\hline Screening & Aztreonam & 94,2 & 26,5 \\
& Ceftriaxone & 99,2 & 11,8 \\
& Cefpodoxime & 95,9 & 11,8 \\
& Ceftazidime & 85,1 & 32,3 \\
& Cefotaxime & 99,2 & 11,8 \\
Confirmatory & Cefpodoxime + clavulanic acid & 76,9 & 100 \\
& Ceftazidime + clavulanic acid & 70,2 & 100 \\
& Cefotaxime + clavulanic acid & 96,7 & 100 \\
\hline
\end{tabular}

Table 3. Potency (MIC50), activity (MIC90) and sensitivity of antimicrobials assayed for $155 \mathrm{ESBL}$ producer isolates

\begin{tabular}{cccc}
\hline Antibiotic & MIC50 & MIC90 & Sensitivity (\%) \\
\hline ATM & 32 & $>256$ & 16.5 \\
COM & 16 & 64 & 46.1 \\
CAZ & 32 & $>128$ & 32.9 \\
CTX & 128 & 256 & 7.4 \\
CRO & 256 & $>256$ & 9.1 \\
IPM & 0.5 & 2 & 100 \\
AMI & 8 & 32 & 86.6 \\
CIP & 16 & $>16$ & 33.8 \\
ERT & 0.12 & 2 & 100 \\
\hline
\end{tabular}

MIC50 = minimum concentration capable to inhibit $50 \%$ of the isolates; MIC90 = minimum concentration capable to inhibit $90 \%$.

with clavulanic acid (CAZ/CAZ+AC) was $89.2 \%$. A comparison of $\mathrm{CPD} / \mathrm{CPD}+\mathrm{AC}$ with cefotaxime and cefotaxime combined with clavulanic acid (CTX/CTX+AC) yielded $97.3 \%$ sensitivity. CAZ/CAZ+AC and CTX/CTX+AC was effective for $99.2 \%$ of the isolates tested. Total sensitivity was only reached when the three combinations were used.

The sensitivity for antimicrobials tested by the agar dilution method for ESBL positive isolates is shown in Table 3. Ertapenem was the antibiotic with the highest potency, followed by imipenem, although they presented the same sensitivity.

\section{Discussion}

ESBLs are the main cause of resistance to beta-lactam antibiotics in members of the Enterobacteriaceae; their biochemistry, genetics and epidemiological characterization have been extensively studied. Due to the clinical importance of the detection of ESBLs, screening and confirmatory methods have been routinely used to investigate the production of these enzymes in E. coli and Klebsiella spp. As their occurrence in other enterobacterias has been increasing, it becomes essential to evaluate their occurrence in this population. 
In our study 121 (24\%) of all the samples and 78\% of previously-screened isolates (as scored by sensitivity to thirdgeneration cephalosporins and aztreonam) were ESBL positive for ESBL by the clavulanic acid inhibition diffusion test. This value is considered high when compared to other reports [8], but is similar to other results found in Brazil, with $29 \%$ of isolates exhibiting ESBLs among all Enterobacteriaceae [18]. This highlights the importance of the investigation of resistance mechanisms in hospitals. The proportion of isolated/ confirmed ESBL producers (78\%) that we found reinforces the need for a confirmatory test in order to distinguish ESBLs from other resistance mechanisms to beta-lactamics.

Klebsiella pneumoniae (67 ESBL positive isolates) was the most common ESBL producer, as described [2]. However E. cloacae (20 ESBL positive isolates) had a higher prevalence than other classic producers (E. coli, 14; K. oxytoca, 3). The same observation was made by Mendes et al. [18]. ESBL positive isolates were found in all species, except for $E$. gergoviae, and the finding of ESBL in several enterobacteria species has already been reported by others $[8,19,20]$.

The occurrence of ESBL in non-E. coli and non-Klebsiella spp. isolates was $21.5 \%$. This is a lower frequency than found for $K$. pneumoniae, which is regarded as the classic ESVL producer. This frequency was similar to that of $K$. oxytoca (21.4\%), and higher than E. coli (7.2\%). Proteus mirabilis ESBL production was found at a similar frequency to $E$. coli production $(8 \%)$. These results are higher than those found in the USA [2, 8], but similar to those obtained in several Latin American countries for Klebsiella spp. and E. coli [21]. Therefore, we conclude that the frequency of ESBL is higher in our geographic region.

Among non-E. coli and non-Klebsiella spp. species, the highest percentage of ESBL-positive isolates was found in Enterobacter (27.1\%), Serratia (20.9\%), Citrobacter (14.3\%) and Morganella (11.8\%). All of the aforementioned harbor chromosomal-inducible AmpC type beta-lactamases. Decreased bacterial membrane permeability to the antimicrobials, associated with the production of other inducible beta-lactamases can mark the presence of ESBL [8,2224]. Since these genera also accounted for a high number of inconclusive samples, it is reasonable to suppose that the occurrence may have been even greater, which points to the necessity of evaluation by molecular biological techniques.

The high number of ESBL isolates resistant to cefoxitine (53\%) can be explained by the high number of isolates among the ESBL positives that harbored chromosomal AmpC, since ESBLs do not confer resistance to that antibiotic. Also, cefoxitine cannot be used alone to distinguish ESBL from AmpC or from other beta-lactamases, because other mechanisms can confer that phenotype, such as mutations in porines alone, or associated with beta-lactamase production [25].

Classic ESBL producers also had positive ESBL isolates resistant to cefoxitine (28 K. pneumoniae, 5 E. coli, $2 \mathrm{~K}$. oxytoca) and $K$. pneumoniae had five inconclusive samples. The recent dissemination of $\mathrm{AmpC}$ plasmidial enzymes in these species could explain these findings, in addition to a possible mutation in porines [25,26]. Moreover, enzymes that mask the presence of ESBL in Enterobacteriaceae may involve betalactamases sensitive to clavulanic acid, and when they are produced in high concentrations they may be capable of reducing the sensitivity to third-generation cephalosporins and aztreonam [3,8,27].

Phenotypic differences were used to distinguish microorganisms that are sensitive to non-ESBL inhibitors from ESBL. In the case of the K1 enzyme of $K$. oxytoca, the property of not conferring resistance to ceftazidime was employed for purposes of distinction. Moreover, K1 only produces resistance to third-generation cephalosporins when it is present in high concentrations, while the concentration of clavulanic acid in the detection test is insufficient to inhibit the enzyme in an effective way [2]. The chromosomal enzymes of Proteus vulgaris were used to differentiate non-ESBL isolates that are sensitive to inhibitors from ESBLs, because they do not confer resistance to aztreonam. The enzymes belonging to group $2 \mathrm{f}$ found in E. cloacae and S. marcescens are differentiated from ESBL producers by their capability to degrade carbapenems $[3,29]$.

Schwaber et al. [8] has reported that there is no need for standardization in the USA for investigation of ESBL in nonE. coli and non-Klebsiella spp. due to the low prevalence of that phenotype $(2.2 \%)$. In our study the prevalence was approximately 10 times higher, justifying the determination of ESBL in non-E. coli and non-Klebsiella spp. species in these hospitals in Curitiba.

Controversies exist regarding the clinical importance of reporting ESBL in chromosomal-inducible beta-lactamaseproducing species that would correspond to most of the nonclassic ESBLs found in our study. The third-generation cephalosporins are considered resistant to that group. Despite this, cefepime may be a therapeutic option for AmpCproducing microorganisms, while ESBL producers are resistant to fourth-generation cephalosporins [29]. In the case of $P$. mirabilis, detection is as important as in E. coli and $K$. pneumoniae, because it does not produce known AmpC chromosomal beta-lactamases [29]. In our study, the incidence of ESBL in P.mirabilis was similar to the incidence in E. coli, but higher numbers of isolates are needed to understand its contribution to the problem. Its detection is epidemiologically important, since the resistance genes are found in conjugative plasmids [24].

Evaluation of the efficiency of the substrate used in the screening and confirmatory tests for ESBL production showed better sensitivity for the antimicrobials ceftriaxone and cefotaxime. When a hierarchy of species is considered, $E$. coli and Klebsiella spp. sensitivities were higher than others. Good results were also found with cefpodoxime and aztreonam, while less satisfactory ones were obtained with ceftazidime, 
especially for non-E. coli and non-Klebsiella species. These results mainly involve the type of enzyme present, which has different affinities for several substrates. In this case, the enzyme has a higher affinity for cefotaxime than for ceftazidime, which is a typical characteristic of the CTX-M beta-lactamases already found in other hospitals in Brazil $[28,30]$; it is considered the dominant type in South America [31].

The sensitivity analysis derived from the addition of clavulanic acid demonstrated the need to associate at least two different combinations of cephalosporins or cephalosporins combined with clavulanic acid in order to obtain a good result. This occurred mainly for non-E. coli and non-Klebsiella species. M'Zali et al. [32] also observed an increase in sensitivity for ESBL detection when more than one substrate was used.

Among the antimicrobials evaluated for minimum inhibitory concentration, the carbapenemics presented the best potency (MIC50), with ertapenem $(0.12 \mu \mathrm{g} / \mathrm{mL})$ being more potent than imipenem $(0.5 \mu \mathrm{g} / \mathrm{mL})$. Kholer et al. [33] indicated that this may be attributable to the high affinity of ertapenem for PBP 3. This value is similar to the affinity of PBP 2 for imipenem or ertapenem. Ertapenem has been recommended for the treatment of infections caused by ESBL-producing bacteria. This antibiotic can be administered in a single daily dose, which reduces cost and facilitates ambulatory treatment. It has also been reported that ertapenem does not present good activity against glucose non-fermenters and does not contribute to the selective pressure responsible for the increase in the prevalence of Pseudomonas spp. and Acinetobacter spp., which are resistant to carbapenemics [3436].

The reduced sensitivity (33.8\%) of ESBL isolates to ciprofloxacin confirms that cross-resistance among betalactamics and quinolones is quite common in enterobacteria. This point is reinforced when one considers that an overall sensitivity of $67.3 \%$ to ciprofloxacine was found among the bacterial isolates in our study. The reason for this association is not well understood, although it is described that patients with quinolone-resistant isolates frequently share prior intensive antibiotic use [37].

Cross-resistance to amikacine was a less common event. In this study, this aminoglycoside presented high potency and sensitivity, following the carbapenems. These results were in accordance with those of other authors [38], despite the fact that amikacine is an antibiotic with high toxicity and its use is quite restricted [39].

MIC50 and MIC90 were high for all the cephalosporins and aztreonam, as expected. The in vitro tests showed sensitivity for some isolates, underlining the need for specific tests for detection of ESBL enzymes to avoid reporting false sensitivity results in routine tests for beta-lactamic antibiotics. That need has been discussed by several authors as essential to guarantee therapy adapted to the patients and yet help in the establishment of adequate control measures [40].
We conclude that there is a necessity to look for ESBL production in non-E. coli and non-Klebsiella spp., at least in South America. Finally, we confirm that cefotaxime is the best antibiotic to date for the screening and confirmation of ESBL in classic ESBL producing as well as other species of Enterobacteriae.

\section{Acknowledgments}

We thank the staff of the Bacteriology Laboratory of the Clinical Hospital of the Federal University of Paraná, for performing primary isolation of the cultures. We also thank Dr. Bruce Koppelman for critical review of this manuscript.

This study was supported in part by Newprov (Curitiba, Brazil), Merck Sharp \& Dohme (São Paulo, Brazil) and NEBaC (Núcleo de Bacteriologia Clínica de Curitiba).

\section{References}

1. Knothe H., Shah P., Kremery V., et al. Transferable resistance to cefotaxime, cefoxitina, cefamandole e cefuroxime in clinical isolates of Klebsiella pneumoniae and Serratia marcescens. Infection 1983; 11: 315-7.

2. Bradford P.A. Extended-spectrum â-lactamases in the $21 \mathrm{st}$ century: caracterization, epidemiology, and detection of this important resistance threat. Clin Microbiol Rev 2001; 14:933-51.

3. Bush K., Jacoby G.A., Medeiros A.A. A functional classification scheme for â-lactamases and its correlation with molecular structures. Antimicrob Agents Chemother 1995;39:1211-33.

4. Bonnet R. Growing Group of Extended-Spectrum â-lactamases: the CTX-M Enzymes. Antimicrob Agents Chemother 2004; $48: 1-14$.

5. Queenan A.M., Foleno B., Gownley C., et al. Effects of inoculum and â-lactamase activity in AmpC - and extended-spectrum â-lactamase (ESBL)-producing Escherichia coli and Klebsiella pneumoniae clinical isolates tested by using NCCLS ESBL methodology. J Clin Microbiol 2004;42:269-75.

6. Gruteke P., Goessens W., Gils J., et al. Patterns of resistance associated with integrons, the extended-spectrum â-lactamase SHV-5 gene, and a multidrug efflux pump of Klebsiella pneumoniae causing a nosocomial outbreak. J Clin Microbiol 2003;41:1161-6.

7. Mulvey M.R., Soule G., Boyd D., et al. Characterization of the first extended-spectrum beta-lactamase-producing Salmonella isolate identified in Canada. J Clin Microbiol 2003;41:460-2.

8. Schwaber M.J., Raney P.M., Rasheed L.K., et al. Utility of NCCLS guidelines for identifying extended-spectrum âlactamases in non-Escherichia coli and non-Klebsiella spp. of Enterobacteriaceae. J Clin Microbiol 2004;42:294-8.

9. Janis E.R. Maintenance by criopreservation . In Janis E.R Maintenace of Microrganisms a Manual of Laboratory Methods. 2.ed. London: Academic Press INC, 1994.

10. Schreckenberber P.C., Janda J.M. Wong J.D., Baron E.J. Algorithims Identification of aerobic Gram-negative bacterias. In Manual of Clinical Microbiology. New York: American Society Microbiology, 2003. 
11. Janda J.M., Abbott S.L., Cheung W.K.W., Henson D.F. Biochemical identification of Citrobacteria in the clinical laboratory. J Clin Microbiol 1994;32:1850-4.

12. O'Hara C.M., Brenner F.W., Miller J.M. Classification, identification, and clinical significance of Proteus, Providencia and Morganella. Clin Microbiol Rev 2000; $13: 534-46$.

13. Sanders W.E.J.R., Sanders C.C. Enterobacter spp: Pathogens poised to flourish at the turn of the century. Clin Microbiol Rev 1997; 10:220-41.

14. National Committee for Clinical Laboratory Standards. Performance standards for antimicrobial disk susceptibility tests. Approved standard. 7.ed. NCCLS 2003; Document M2-A8. NCCLS. Wayne. PA.

15. Clinical Laboratory Standard Institute (CLSI). Performance standards for antimicrobial susceptibility testing. Wayne, PA: CLSI 2005.

16. National Committee for Clinical Laboratory Standards. Methods for dilution antimicrobial susceptibility tests for bacteria that grow aerobically. Approved standard. 5.ed., Document M7A6. Wayne, PA: NCCLS 2003.

17. Podschun R., Ullmann, A. Klebsiella spp. as nosocomial pathogens: epidemiology, taxonomy, typing methods, and pathogenicity factors. J Clin Microbiol 1998;11:589-603.

18. Mendes C., Hsiung A., Kiffer C., et al. Evaluation of the in vivo Activity of 9 Antimicrobials Against Bacterial Strains Isolated from Patients in Intensive Care Units in Brazil: MYSTIC Antimicrobial Surveillance Program . Braz J Infect Dis 2000;4:236-44.

19. Pagani L., Migliavacca R., Pallecchi L., et al. Emerging extendedspectrum â-lactamases in Proteus mirabilis. J Clin Microbiol 2002; 40:1549-52.

20. Champs C., Bonnet R., Sirot D., et al. Clinical relevance of Proteus mirabilis in hospital patients: a two year survey. $\mathrm{J}$ Antimicrob Chemoter 2000;45:537-9.

21. Sader H.S., Gales A.C., Pfaller M.A., et al. Pathogen frequency and resistance patterns in brazilian hospitals: summary of results from three years of the SENTRY antimicrobial surveillance program. Braz J Infect Dis 2001;5:200-14.

22. Tenover F.C., Mohammed M.J., Gorton T.S., Dembek Z.F. Detection and reporting of organisms producing extendedspectrum â-lactamases: survey of laboratories in Connecticut. J Clin Microbiol 1999;37:4065-70.

23. Varela C., Oliver A., Coque T.M., et al. Prevalence of extendedspectrum â-lactamases in group-1 â-lactamases-producing isolates. Clin Microbiol Infect 2001;7:278-82.

24. Thomson K.S. Controversies about extended-spectrum and AmpC beta-lactamases. Em Infect Dis 2001;7:333-6.

25. Yan J.J., Ko W., Wu H., et al. Complexity of Klebsiella pneumoniae Isolates Resistant to Both Cephamycins and Extended-Spectrum Cephalosporins at a Teaching Hospital in Taiwan. J Clin Microbiol 2004;42:5337-40.
26. Coudron P.E., Hanson N.D., Climo M.W. Occurrence of extended-spectrum â-lactamase in bloodstream isolates of Klebsiella pneumoniae: isolates harbor plasmid-mediate FOX5 and ACT-1 AmpC â-lactamases. J Clin Microbiol 2003; $41: 772-7$

27. Livermore D.M. â-Lactamases in laboratory and clinical resistance. Clin Microbiol Rev 2001;8:557-84.

28. Bonnet R., Dutour C., Sampaio J.L.M., et al. Novel Cefotaximase (CTX-M-16) with Increased Catalytic Efficiency Due to Substitution Asp-240? Gly. Antimicrob Agents Chemother 2001;45:2269-75.

29. Livermore D.M. â-Lactamases in laboratory and clinical resistance. Clin Microbiol Rev 1995;8:557-84.

30. Bonnet R., Sampaio J.L.M., Labia R., et al. A novel CTX-M âlactamase (CTX-M-8) in cefotaxime-resistant Enterobacteriaceae isolated in Brazil. Antimicrob Agents Chemother 2000; 44 : 1936-42.

31. Livermore D.M., Brown D.F.J. Detection of â-lactamase-mediated resistance. J Antimicrobial Chemoter 2001;48:59-64.

32. M`Zali F.H., Chanawong A., Kerr K.G., et al. Detection of extended-spectrum â-lactamases in members of the family Enterobacteriaceae: comparison of the MAST DD test, the double disk and the E-test ESBL, J Antimicrobial Chemoter 2000; $45: 881-5$.

33. Kholer J., Dorso K.L., Young K., et al. In vitro Activies of the Potent, Broad-Spectrum Carbapenem MK-0826 (L-749,345) against Broad-Spectrum â-lactamases and Extended-spectrum âlactamase-producing Klebsiella pneumoniae and Escherichia coli Clinical Isolates. Antimicrob Agents Chemother 1999;43:1170-6.

34. Livermore D.M., Sefton A.M., Scott G.M. Properties e Potential of Ertapenem. J Antimicrobial Chemoter 2003;52:331-44.

35. Livermore D.M., Oakton K.J., Carter M.W., Warner M. Activity of Ertapenem (MK-0826) versus Enterobacteriaceae with Potent â-lactamases. Antimicrob Agents Chemother 2001;45:2831-7.

36. Fuchs P.C., Barry A.L., Brown S.D. In vitro Activities of Ertapenem (MK-0826) agains Clinical Bacterial Isolates from 11 North American Medical Centers. Antimicrob Agents Chemother 2001; 45:1915-8.

37. Patterson J.E. Antibiotic utilization: is there an effect on antimicrobial resistance? Chest 2001;119:426-30.

38. Shukia I., Tiwari R., Agraval M. Prevalence of extended spectrum â-lactamase producing Klebsiella pneumoniae in a tertiary care hospital. Indian J of Med Microbiol 2004;22:87-91.

39. Tavares W. Aminoglicosideos In: Tavares W. Manual de antibiótico e quimioterápicos antiinfecciosos. São Paulo: Atheneu, 2001.

40. Essack S.Y. Laboratory detection of extended-spectrum âlactamases (ESBLs) The need for a realiable, reproducible method. Diagn Microbial Infec Dis, 2000;37:2000. 